\title{
ON THE USE OF STATE CONSTRAINTS IN OPTIMAL CONTROL OF SINGULAR PDES
}

\author{
Christian Clason* Barbara Kaltenbacher ${ }^{\dagger}$
}

June 18, 2012

\begin{abstract}
We consider optimal control of nonlinear partial differential equations involving potentially singular solution-dependent terms. Singularity can be prevented by either restricting controls to a closed admissible set for which well-posedness of the equation can be guaranteed, or by explicitly enforcing pointwise bounds on the state. By means of an elliptic model problem, we contrast the requirements for deriving existence of solutions and first order optimality conditions for both the control-constrained and the state-constrained formulation. Our analysis as well as numerical tests illustrate that control constraints lead to severe restrictions on the attainable states, which is not the case for state constraints.
\end{abstract}

\section{INTRODUCTION}

Many nonlinear models in science and engineering involve solution-dependent terms that can become singular, signaling failure of the model or actual physical blow-up. This introduces difficulties for the optimal control of such equations, since existence of a solution to the state equation can be guaranteed a priori only for controls which are small in some norm. Ensuring well-posedness of the control-to-state mapping thus requires imposing such a bound as a control constraint. The purpose of this work is to demonstrate that this is an overly restrictive approach that can prevent even approximate attainment of the prescribed target, and that this can be avoided by incorporating explicit pointwise bounds as state constraints into the optimal control problem.

\footnotetext{
*Institute for Mathematics and Scientific Computing, University of Graz, Heinrichstrasse 36, A-8010 Graz, Austria (christian.clason@uni-graz.at)

${ }^{\dagger}$ Institute of Mathematics, Alpen-Adria Universität Klagenfurt, Universitätsstrasse 65-67 A-9020 Klagenfurt, Austria (barbara.kaltenbacher@uni-klu.ac.at)
} 
We focus on a simple model problem which allows explicit calculation of bounds (and is of practical relevance in itself), although the approach is applicable for a wide range of nonlinear models with potential degeneracy. Consider

$$
\left\{\begin{array}{cl}
-\Delta y=-\frac{\beta u}{(1+y)^{2}} & \text { on } \Omega, \\
y=0 & \text { on } \partial \Omega
\end{array}\right.
$$

for convex $\Omega \subseteq \mathbb{R}^{n}, n \in\{1,2,3\}$ (typically $n=2$ ), with $C^{1}$ boundary $\partial \Omega$. This equation models the deflection of an idealized electrostatically actuated microelectromechanical systems (MEMS), where $y$ is the mechanical displacement, $u$ is the reciprocal of the dielectric constant and $\beta$ is a dimensionless number proportional to the applied voltage [Pelesko 2001/o2], [Pelesko and Bernstein 2003]. The case $y(x)=-1$ corresponds to the "pull-in" instability, in which the applied voltage leads to a sufficiently large deflection of the membrane for it to touch the ground plate, possibly damaging the device. Motivated by the problem of optimal design of dielectric properties of the membrane, we consider the control problem

$$
\left\{\begin{array}{l}
\min _{y, u} \frac{1}{2}\left\|y-y_{d}\right\|_{L^{2}(\Omega)}^{2}+\frac{\alpha}{2}\|u\|_{L^{2}(\Omega)}^{2} \\
-\Delta y=-\frac{\beta u}{(1+y)^{2}},\left.\quad y\right|_{\partial \Omega}=0,
\end{array}\right.
$$

where $y_{d}$ is a prescribed displacement under a given voltage. To prevent the "pull-in" instability, we contrast the use of two different constraints:

- control constraints: $\|\mathrm{u}\|_{\mathrm{L}^{2}(\Omega)} \leqslant \mathrm{M}_{\mathfrak{u}}$, where $\mathrm{M}_{\mathrm{u}}$ is chosen small enough so that $\|\mathrm{y}\|_{\mathrm{C}(\Omega)}<$ 1 , and

- state constraints: $-\mathrm{y}(x) \leqslant M_{y}$ for all $x \in \Omega$, where $M_{y}<1$.

While the former approach allows application of standard techniques since the control-tostate mapping $u \mapsto y$ is well-defined for all admissible $u$, this is not the case for the latter. The main difficulties are that (1.1) is not of monotone type, and that the reduced formulation is not well-defined for all $u \in \mathcal{U}$, due to potential degeneracy of the equation. Indeed it has been shown (Theorem 3.1 in [Pelesko 2001/02]) that (1.1) is not solvable for sufficiently large controls $u$; hence the control or state constraints are vital for guaranteeing that the PDE is solvable at all. Such constraints are also necessary to ensure well-posedness of algorithms for the numerical solution of the optimal control problem (1.2). Correspondingly, the crucial step for deriving optimality conditions in both approaches is proving well-posedness of the linearized equation, for which the condition in the state-constrained case will turn out to be less restrictive.

Optimal control of elliptic partial differential equations subject to control constraints is by now classical (cf., e.g., [Tröltzsch 2010] and the literature cited therein). The literature on state constraints is now similarly extensive, and we only refer to the standard works [Alibert and Raymond 1998; Casas 1986; Bergounioux and Kunisch 1997] (as well as the aforementioned 
monograph). Constraints for discontinuous and possibly unbounded states were considered in [Schiela 2009], although their objective is different from ours (missing regularity of the state as opposed to pointwise singularity of the equation here) and they consider linear PDE constraints only. Control of non-monotone nonlinear elliptic systems without state constraints are discussed in [Lions 1985] using a penalization approach. Non-monotone systems with state constraints were treated in [Bonnans and Casas 1989] for the case of right-hand-side controls.

Although the results on optimal control of the MEMS model problem are new in themselves, the use of state constraints for the purpose of preventing singularity of the state equation is the main novel idea of this paper. It is expected to have many practically relevant applications for the control of potentially singular PDEs, such as the Westervelt and the Kuznetsov equation in nonlinear acoustics [Clason et al. 2009], Euler equations with general pressure laws in isentropic gas dynamics [Chen 2010], or the Richards equation modeling flow in unsaturated porous media [Pop and Schweizer 2011], to name just a few examples.

Remark 1.1. Although in the considered application, the physically relevant regime requires $\beta \geqslant 0$ and $1 \geqslant u>0$, we do not enforce these constraints in order to focus on the use of control or state constraints to prevent singularity of the state equation, since the main purpose of this model is to illustrate and contrast the general approaches.

This paper is organized as follows. In the remainder of this section, we introduce some common notation for both approaches. In section 2, we discuss the control-constrained optimal control problem, for which we derive sufficient conditions for well-posedness and differentiability of the control-to-state mapping in section 2.1. These are used in section 2.2 to obtain first order optimality conditions. The case of state constraints is considered in section 3, where we consider the state equation as a nonlinear equality constraint, for which we derive continuity and differentiability in Section 3.1. The corresponding optimality system is discussed in section 3.2. Section 4 contrasts both approaches for a simple numerical example.

In the following, we denote the unconstrained control space by

$$
\mathcal{U}:=\mathrm{L}^{2}(\Omega)
$$

and the unconstrained state space by

$$
y:=H^{2}(\Omega) \cap H_{0}^{1}(\Omega) \hookrightarrow C_{0}(\Omega),
$$

where the embedding is continuous and compact. Under the assumptions on $\Omega$ (convexity, $\mathrm{C}^{1}$ boundary), the inverse Laplacian is a continuous operator from $\mathrm{L}^{2}(\Omega)$ to $\mathrm{H}^{2}(\Omega)$ as well as from $\mathrm{W}^{-1, p}(\Omega)$ to $\mathrm{W}^{1, p}(\Omega)$ for all $2<\mathrm{p}<\infty$ (see, e.g., [Troianiello 1987], Th. 2.24 and Th. 3.16(iv), respectively). Due to the compact embedding of $W^{1, p}$ in $C_{0}(\Omega)$ for $p>n$, we can make use of the lifting from $\mathrm{L}^{2}(\Omega)$ to $\mathrm{C}_{0}(\Omega)$ via the inverse Laplacian with Dirichlet conditions, which is a continuous operator, i.e., there exists a $C_{L}>0$ such that for all $v \in$ $\mathrm{L}^{2}(\Omega)$,

$$
\left\|(-\Delta)^{-1} f\right\|_{C(\Omega)} \leqslant C_{L}\|f\|_{L^{2}(\Omega)}
$$


holds.

For given $M_{\mathfrak{u}}>0$, we define the admissible control space

$$
\mathcal{U}_{M}:=\left\{u \in \mathcal{U}:\|u\|_{\mathrm{L}^{2}(\Omega)} \leqslant M_{\mathfrak{u}}\right\}
$$

and for $M_{y}<1$, the admissible state space

$$
y_{M}:=\left\{y \in y:-y(x) \leqslant M_{y} \text { for all } x \in \Omega\right\} .
$$

Remark 1.2. The results below remain valid for any cost functional $J(u, y)$ that is bounded from below, coercive with respect to $u$ (this condition may be omitted in the control-constrained case), weakly lower semicontinuous, and differentiable on $\mathcal{U} \times y$.

\section{CONTROL CONSTRAINTS}

For the sake of comparison, we first consider the control-constrained optimal control problem

$\left(\mathcal{P}_{\mathrm{cc}}\right)$

$$
\left\{\begin{array}{l}
\min _{y \in y, u \in U} \frac{1}{2}\left\|y-y_{d}\right\|_{L^{2}(\Omega)}^{2}+\frac{\alpha}{2}\|u\|_{L^{2}(\Omega)}^{2} \\
-\Delta y=-\frac{\beta u}{(1+y)^{2}} \\
\|u\|_{L^{2}(\Omega)}^{2} \leqslant M_{u}^{2}
\end{array}\right.
$$

where $M_{\mathfrak{u}}$ is chosen sufficiently small to obtain well-posedness of the state equation.

\subsection{STATE EQUATION}

We first derive sufficient conditions on $u$ such that (1.1) has a unique solution. The proof technique is chosen to yield explicit bounds which can be used as control constraints.

Theorem 2.1. There exists a constant $M_{\mathfrak{u}}>0$ such that for all $u \in \mathcal{U}_{M}$, there exists a unique solution $y \in y$ to (1.1).

Proof. We use Banach's fixed point theorem with the fixed point operator $T: W \rightarrow W$, $v \mapsto \mathrm{T} v:=\mathrm{y}$, where $\mathrm{y}$ solves

$$
\left\{\begin{aligned}
-\Delta y & =-\frac{\beta u}{(1+v)^{2}} & & \text { on } \Omega, \\
y & =0 & & \text { on } \partial \Omega,
\end{aligned}\right.
$$

and

$$
W=\left\{v \in y:\|v\|_{C(\Omega)} \leqslant \bar{m}\right\}
$$


for some $\bar{m} \in(0,1)$. To show that $T$ is a self-mapping, consider an arbitrary $v \in W$. Then we have

$$
\|\mathrm{T} v\|_{\mathrm{C}(\Omega)} \leqslant \mathrm{C}_{\mathrm{L}}|\beta|\left\|\frac{u}{(1+v)^{2}}\right\|_{\mathrm{L}^{2}(\Omega)} \leqslant \frac{\mathrm{C}_{\mathrm{L}}|\beta|}{(1-\overline{\mathrm{m}})^{2}}\|u\|_{\mathrm{L}^{2}(\Omega)}
$$

where $C_{L}$ is the constant in (1.3), and the right hand side is not larger than $\bar{m}$ if

$$
\|u\|_{L^{2}(\Omega)} \leqslant \frac{\bar{m}(1-\bar{m})^{2}}{C_{L}|\beta|}
$$

Contractivity of $\mathrm{T}$ follows from the fact that for any $v, w \in \mathrm{W}$, there holds

$$
\begin{aligned}
\|\mathrm{T} v-\mathrm{T} w\|_{\mathrm{C}(\Omega)} & \leqslant \mathrm{C}_{\mathrm{L}}|\beta|\left\|\left(\frac{1}{(1+v)^{2}}-\frac{1}{(1+w)^{2}}\right) \mathrm{u}\right\|_{\mathrm{L}^{2}(\Omega)} \\
& \leqslant \mathrm{C}_{\mathrm{L}}|\beta|\left\|\left(\frac{1}{(1+v)^{2}(1+w)}+\frac{1}{(1+w)^{2}(1+v)}\right)(v-w) \mathrm{u}\right\|_{\mathrm{L}^{2}(\Omega)} \\
& \leqslant \frac{2 \mathrm{C}_{\mathrm{L}}|\beta|}{(1-\overline{\mathrm{m}})^{3}}\|\mathrm{u}\|_{\mathrm{L}^{2}(\Omega)}\|v-w\|_{\mathrm{C}(\Omega)} .
\end{aligned}
$$

Hence contractivity holds if

$$
\|u\|_{L^{2}(\Omega)}<\frac{(1-\bar{m})^{3}}{2 C_{L}|\beta|}
$$

The maximum over $\bar{m} \in(0,1)$ of the minimum of the two right hand sides in (2.2) and (2.3) can be found by equilibrating $\frac{\bar{m}(1-\bar{m})^{2}}{C_{L}|\beta|}=\frac{(1-\bar{m})^{3}}{2 C_{L}|\beta|}$, which yields the optimal bound

$$
\overline{\mathrm{m}}=\frac{1}{3} \text {. }
$$

Therefore, if

$$
\|u\|_{L^{2}(\Omega)} \leqslant M_{u}:=\frac{4}{27 C_{L}|\beta|}
$$

holds, the operator T has a unique fixed point $y^{*} \in W$ which by construction satisfies (1.1).

Remark 2.2. For $\Omega=(0,1)^{2}$ and $\beta=1$, we can explicitly calculate

$$
M_{\mathfrak{u}}=\frac{4}{27} \approx 0.14815
$$

Remark 2.3. Even if we use a compactness argument instead of contractivity for showing existence, condition (2.2) for the self-mapping property remains. The right hand side in (2.2) is maximal for $\bar{m}=\frac{1}{3}$. Any different $\bar{m}$ (e.g., closer to one that increases the bound on $\|v\|_{C(\Omega)}$ in (2.1)) actually makes the control bound in (2.2) more restrictive and therefore increases the optimal cost function value in $\left(\mathcal{P}_{\mathfrak{c c}}\right)$, signifying even poorer attainment of the target. 
We can now introduce the control-to-state mapping

$$
S: \mathcal{U}_{M} \rightarrow y, \quad u \mapsto y \text { solving (1.1), }
$$

which by Theorem 2.1 is well-defined for sufficiently small $M_{\mathfrak{u}}$ satisfying (2.4). We next show continuity of $S$.

Theorem 2.4. There exists a constant $M_{\mathfrak{u}}>0$ such that the control-to-state mapping $S: \mathfrak{U}_{M} \rightarrow$ $y$ is Lipschitz continuous and weakly continuous.

Proof. The difference $\tilde{y}-y$ between two solutions $\tilde{y}=S(\tilde{u})$ and $y=S(u)$ satisfies

$$
\left\{\begin{aligned}
-\Delta(\tilde{y}-y)-\frac{\beta \tilde{u}(2+\tilde{y}+y)}{(1+y)^{2}(1+\tilde{y})^{2}}(\tilde{y}-y) & =-\frac{\beta}{(1+y)^{2}}(\tilde{u}-u) & & \text { on } \Omega, \\
\tilde{y}-y & =0 & & \text { on } \partial \Omega
\end{aligned}\right.
$$

hence

$$
\|\Delta(\tilde{y}-y)\|_{L^{2}(\Omega)} \leqslant \frac{2|\beta|}{(1-\bar{m})^{3}}\|\tilde{u}\|_{L^{2}(\Omega)}\|\tilde{y}-y\|_{C(\Omega)}+\frac{|\beta|}{(1-\bar{m})^{2}}\|\tilde{u}-u\|_{L^{2}(\Omega)}
$$

where we have used the fact that $\tilde{y}, y \in W$, with $W$ defined as in (2.1). Thus we arrive at

$$
\|\Delta(\tilde{y}-y)\|_{L^{2}(\Omega)} \leqslant \mathrm{L}\left(\overline{\mathrm{m}}, M_{\mathfrak{u}}\right)\|\tilde{u}-\mathfrak{u}\|_{\mathrm{L}^{2}(\Omega)}
$$

where

$$
L\left(\bar{m}, M_{u}\right):=\left\{1-\frac{2 C_{L}|\beta| M_{u}}{(1-\bar{m})^{3}}\right\}^{-1} \frac{|\beta|}{(1-\bar{m})^{2}},
$$

provided (2.3) holds, which implies that the expression in braces is positive. This shows continuity with Lipschitz constant $\mathrm{L}\left(\overline{\mathrm{m}}, \mathrm{M}_{\mathfrak{u}}\right)$.

To see weak continuity we use a subsequence-subsequence argument. Let $\left(u_{n}\right)_{n \in \mathbb{N}} \subseteq \mathcal{U}_{M}$ be an arbitrary sequence converging weakly to $u^{*}$ in $L^{2}(\Omega)$. Then $u^{*} \in \mathcal{U}_{M}$, and the sequence $\left(S\left(u_{n}\right)\right)_{n \in \mathbb{N}} \subseteq y$ is bounded in $y\left(\right.$ by $|\beta|(1-\bar{m})^{-2} \sup _{n \in \mathbb{N}}\left\|u_{n}\right\|_{L^{2}(\Omega)}$ with $\bar{m}$ as in the proof of Theorem 2.1). Therefore any subsequence of $\left(S\left(u_{n}\right)\right)_{n \in \mathbb{N}}$ has a weakly convergent subsequence. Moreover, the limit $y^{*}$ of any weakly convergent subsequence of $\left(y_{n}\right)_{n \in \mathbb{N}}=\left(S\left(u_{n}\right)\right)_{n \in \mathbb{N}}-$ which by compactness of the embedding $y \rightarrow C_{0}(\Omega)$ contains a subsequence, again denoted by $\left(y_{n}\right)_{n \in \mathbb{N}}$, converging strongly in $C_{0}(\Omega)$ to $y^{*}$ - has to solve (1.1) with $u=u^{*}$. Indeed, for any $v \in \mathrm{L}^{2}(\Omega)$ we have

$$
\begin{aligned}
0= & \int_{\Omega}\left(\Delta y_{n}-\frac{\beta u_{n}}{\left(1+y_{n}\right)^{2}}\right) v d x=\int_{\Omega}\left(\Delta y^{*}-\frac{\beta u^{*}}{\left(1+y^{*}\right)^{2}}\right) v d x \\
& +\int_{\Omega}\left(\Delta y_{n}-\Delta y^{*}\right) v d x-\int_{\Omega}\left(\frac{1}{\left(1+y_{n}\right)^{2}}-\frac{1}{\left(1+y^{*}\right)^{2}}\right) \beta u_{n} v d x \\
& -\int_{\Omega}\left(u_{n}-u^{*}\right) \frac{\beta v}{\left(1+y^{*}\right)^{2}} d x,
\end{aligned}
$$


where all terms in the last two lines go to zero as $n \rightarrow \infty$ : the first one by weak convergence of $y_{n}$ in $y$, the second one by strong convergence of $y_{n}$ in $C_{0}(\Omega)$ and boundedness of $u_{n}$ in $\mathrm{L}^{2}(\Omega)$, and the third one by weak convergence of $u_{n}$ in $\mathrm{L}^{2}(\Omega)$. Therefore, by uniqueness in Theorem 2.1, $\mathrm{y}^{*}$ coincides with $\mathrm{S}\left(\mathrm{u}^{*}\right)$.

To show differentiability, we need the well-posedness of the linearized state equation at $\bar{u} \in \mathcal{U}_{M}, \bar{y} \in y_{:}$

$$
\left\{\begin{aligned}
-\Delta y-\frac{2 \beta \bar{u}}{(1+\bar{y})^{3}} y=f & \text { on } \Omega, \\
y=0 & \text { on } \partial \Omega,
\end{aligned}\right.
$$

for given $f \in L^{2}(\Omega)$.

Lemma 2.5. For all $\overline{\mathrm{u}} \in \mathcal{U}_{\mathrm{M}}, \overline{\mathrm{y}}=\mathrm{S}(\overline{\mathrm{u}})$, and all $\mathrm{f} \in \mathrm{L}^{2}(\Omega)$, the linearized state equation (2.5) has a unique solution $y \in \mathcal{y}$, which depends continuously on $f$.

Proof. Note that the coefficient $a:=-\frac{2 \beta \bar{u}}{(1+\bar{y})^{3}}$ typically has the wrong sign and is not in $\mathrm{L}^{\infty}(\Omega)$, so the Lax-Milgram Lemma does not apply directly to (2.5), but rather a fixed point argument similar to the one in the proof of Theorem 2.1 has to be used. We refer to [Colonius and Kunisch 1986] but nevertheless provide the full proof for the setting relevant here.

Consider the fixed point operator mapping $v$ to $\tilde{T} v:=y$ defined as the solution to

$$
\left\{\begin{aligned}
-\Delta y & =-a v+f & & \text { on } \Omega, \\
y & =0 & & \text { on } \partial \Omega .
\end{aligned}\right.
$$

Due to the estimate

$$
\|\Delta \tilde{\mathrm{T}} v\|_{\mathrm{L}^{2}(\Omega)} \leqslant \mathrm{C}_{\mathrm{L}}\|\mathrm{a}\|_{\mathrm{L}^{2}(\Omega)}\|\Delta v\|_{\mathrm{L}^{2}(\Omega)}+\|\mathrm{f}\|_{\mathrm{L}^{2}(\Omega)},
$$

the operator $\tilde{\mathrm{T}}$ is a self-mapping on

$$
\tilde{W}=\left\{v \in y:\|\Delta v\|_{L^{2}(\Omega)} \leqslant R\right\}
$$

for any fixed $R>0$, as long as

$$
\mathrm{C}_{\mathrm{L}}\|\mathrm{a}\|_{\mathrm{L}^{2}(\Omega)}<1
$$

is satisfied (which can be guaranteed by $\|\bar{u}\|_{L^{2}(\Omega)} \leqslant M_{u}$ as in (2.3)) and $\|f\|_{L^{2}(\Omega)} \leqslant(1-$ $\left.C_{L}\|\mathrm{a}\|_{\mathrm{L}^{2}(\Omega)}\right) \mathrm{R}=\mathrm{r}$. Condition (2.7) also yields contractivity via the estimate

$$
\|\Delta(\tilde{\mathrm{T}} v-\tilde{\mathrm{T}} w)\|_{\mathrm{L}^{2}(\Omega)} \leqslant \mathrm{C}_{\mathrm{L}}\|\mathrm{a}\|_{\mathrm{L}^{2}(\Omega)}\|\Delta(v-w)\|_{\mathrm{L}^{2}(\Omega)},
$$

hence $\tilde{T}$ has a unique fixed point $y \in W$. Using estimate (2.6) with $y$ in place of $v$ and $\tilde{T} v$, we deduce that the operator $\tilde{S}$ mapping $f$ to a solution of $(2.5)$ is well-defined and bounded from $\mathrm{L}^{2}(\Omega)$ to $y$ - first, on the ball of radius $r$, and hence by linearity on $\mathrm{L}^{2}(\Omega)$. 
Finally, we address differentiability of the control-to-state mapping.

Theorem 2.6. The control-to state mapping $S: \mathcal{U}_{M} \rightarrow y$ is Fréchet differentiable, with derivative $\mathrm{S}^{\prime}(\mathrm{u})(\tilde{\mathrm{u}}-\mathrm{u})$ given by the solution $w \in \mathrm{y}$ to

$$
\left\{\begin{aligned}
-\Delta w-\frac{2 \beta u}{(1+y)^{3}} w & =-\frac{\beta}{(1+y)^{2}}(\tilde{u}-u) & & \text { on } \Omega, \\
w & =0 & & \text { on } \partial \Omega
\end{aligned}\right.
$$

where $\mathrm{y}=\mathrm{S}(\mathrm{u})$.

Proof. By Lemma 2.5, a unique solution $w \in y$ of (2.8) exists for any $u \in \mathcal{U}_{M}$. To show Fréchet differentiability of $S$, we use the fact that $z:=\tilde{y}-y-w$ solves

$$
\left\{\begin{array}{rlrl}
-\Delta z-\frac{2 \beta u}{(1+y)^{3}} z & =\frac{2 \beta}{(1+y)^{3}}(\tilde{u}-u)(\tilde{y}-y) & \\
& -\frac{\beta \tilde{u}(3+y-\tilde{y})}{(1+y)^{3}(1+\tilde{y})^{2}}(\tilde{y}-y)^{2} & \text { on } \Omega, \\
z=0 & \text { on } \partial \Omega,
\end{array}\right.
$$

to estimate

$$
\begin{aligned}
\|\Delta(\tilde{y}-y-w)\|_{L^{2}(\Omega)} \leqslant & \frac{2|\beta|}{(1-\bar{m})^{3}}\|u\|_{L^{2}(\Omega)}\|\tilde{y}-y-w\|_{C(\Omega)} \\
& +\frac{2|\beta|}{(1-\bar{m})^{3}}\|\tilde{u}-u\|_{L^{2}(\Omega)}\|\tilde{y}-y\|_{C(\Omega)} \\
& +\frac{|\beta|(3+2 \bar{m})}{(1-\bar{m})^{5}}\|\tilde{u}\|_{L^{2}(\Omega)}\|\tilde{y}-y\|_{C(\Omega)}^{2}
\end{aligned}
$$

Hence

$$
\|\Delta(\tilde{y}-y-w)\|_{L^{2}(\Omega)} \leqslant K\left(\bar{m}, M_{u}\right)\|\tilde{u}-u\|_{L^{2}(\Omega)}^{2}
$$

with

$$
\begin{aligned}
& K\left(\bar{m}, M_{u}\right):=\left\{1-\frac{2 C_{L}|\beta|}{(1-\bar{m})^{3}} M_{u}\right\}^{-1} \\
& \cdot \frac{C_{L} L\left(\bar{m}, M_{u}\right)|\beta|\left((3+2 \bar{m}) M_{u} C_{L} L\left(\bar{m}, M_{u}\right)+2(1-\bar{m})^{2}\right)}{(1-\bar{m})^{5}},
\end{aligned}
$$

the expression in braces being positive again due to the choice (2.3) of $M_{\mathfrak{u}}$. 


\subsection{CONTROL PROBLEM}

Since the state equation is well-posed for every $u \in \mathcal{U}_{M}$, we can use the control-to-state mapping $S: \mathcal{U}_{M} \rightarrow y$ to obtain the reduced problem

$$
\min _{u \in \mathcal{U}_{M}} \frac{1}{2}\left\|S(u)-y_{d}\right\|_{L^{2}(\Omega)}^{2}+\frac{\alpha}{2}\|u\|_{L^{2}(\Omega)}^{2} .
$$

Since $\mathcal{U}_{M}$ is nonempty and weakly sequentially compact, and $S$ is weakly continuous for all $u \in \mathcal{U}_{M}$ by Theorem 2.4, we obtain the existence of a minimizer $u^{*} \in \mathcal{U}_{M}$ by standard arguments (see, e.g. [Tröltzsch 2010]).

First order necessary optimality conditions follow from standard arguments as well. For a local minimizer $u^{*}$ of $\left(\mathcal{P}_{\mathrm{cc}}^{\prime}\right)$ and $y^{*}:=S\left(u^{*}\right) \in y$, we can introduce the adjoint state $p^{*} \in y$ solving

$$
\left\{\begin{aligned}
-\Delta p^{*}-\frac{2 \beta u^{*}}{\left(1+y^{*}\right)^{3}} p^{*} & =-\left(y^{*}-y_{d}\right) & & \text { on } \Omega, \\
p^{*} & =0 & & \text { on } \partial \Omega,
\end{aligned}\right.
$$

since the linearized state equation is self-adjoint and is surjective by Lemma 2.5. Furthermore, a Slater condition is trivially satisfied for the inequality constraint $\left\|u^{*}\right\|_{L^{2}(\Omega)}^{2} \leqslant M_{u}^{2}$ (take $u=0$ ). From, e.g., Proposition 3.2 in [Bonnans and Shapiro 2000], we thus deduce the existence of a corresponding Lagrange multiplier $\lambda^{*} \in \mathbb{R}$ and hence the following optimality system.

Theorem 2.7. Let $\mathrm{u}^{*} \in \mathcal{U}_{\mathrm{M}}$ be a local minimizer of $\left(\mathcal{P}_{\mathrm{cc}}^{\prime}\right), \mathrm{y}^{*}:=\mathrm{S}\left(\mathrm{u}^{*}\right) \in \mathrm{y}$, and $\mathrm{p}^{*} \in \mathrm{y}$ satisfy (2.9). Then there exists $\lambda^{*} \in \mathbb{R}$ satisfying $\lambda^{*} \geqslant 0$ and

$\left(\mathrm{OS}_{\mathrm{cc}}\right)$

$$
\left\{\begin{array}{c}
\alpha u^{*}-\frac{\beta}{\left(1+y^{*}\right)^{2}} p^{*}=0, \\
\lambda^{*}\left(\left\|u^{*}\right\|_{L^{2}(\Omega)}^{2}-M_{u}^{2}\right)=0 .
\end{array}\right.
$$

\section{STATE CONSTRAINTS}

Consider now the state-constrained optimal control problem

$\left(\mathcal{P}_{\text {sc }}\right)$

$$
\left\{\begin{array}{l}
\min _{y \in \mathcal{y}, u \in U} \frac{1}{2}\left\|y-y_{d}\right\|_{L^{2}(\Omega)}^{2}+\frac{\alpha}{2}\|u\|_{L^{2}(\Omega)}^{2} \\
-\Delta y=-\frac{\beta u}{(1+y)^{2}}, \\
-y(x) \leqslant M_{y} \quad \text { for all } x \in \Omega,
\end{array}\right.
$$

for given $M_{y}<1$. Since the state equation is not well-posed for every $u \in \mathcal{U}$, we cannot use the results of Section 2.1 but argue directly. We define

$$
\mathrm{G}: U \times \tilde{y}_{M} \rightarrow \mathrm{L}^{2}(\Omega), \quad \mathrm{G}(\mathrm{u}, \mathrm{y})=-\Delta \mathrm{y}+\frac{\beta \mathrm{u}}{(1+y)^{2}}
$$


where $\tilde{y}_{M}:=\left\{y \in y:-y(x) \leqslant \frac{1}{2}\left(M_{y}+1\right), x \in \Omega\right\} \supset y_{M}$. The next section is concerned with continuity and differentiability of $\mathrm{G}$.

\subsection{STATE EQUATION}

We first note that for all $y \in y_{M}$, the term $\frac{\beta}{(1+y)^{2}}$ is uniformly bounded by $\bar{m}:=|\beta|(1-$ $\left.M_{y}\right)^{-2}$. Due to the linearity of $G$ in $u$, it therefore immediately follows that $G$ is continuously differentiable with respect to $u$ for all $(\bar{u}, \bar{y}) \in \mathcal{U} \times y_{M}$, with Fréchet-derivative in direction $h \in \mathcal{U}$ given by

$$
\mathrm{G}_{\mathrm{u}}(\overline{\mathrm{u}}, \overline{\mathrm{y}}) \mathrm{h}=\frac{\beta}{(1+\overline{\mathrm{y}})^{2}} \mathrm{~h} .
$$

To show differentiability with respect to $y$, we consider

$$
\Psi: y(x) \mapsto \frac{\beta}{(1+y(x))^{2}} .
$$

as a Nemytskii operator from $y_{M} \subset L^{\infty}(\Omega)$ to $L^{\infty}(\Omega)$ generated by the mapping

$$
\psi:\left[-M_{y}, \infty\right) \times \Omega \rightarrow \mathbb{R}, \quad \psi(t, x):=\frac{\beta}{(1+t)^{2}} .
$$

Since $\psi$ is measurable for fixed $t \geqslant-M_{y}>-1$ and continuous in $t$ uniformly for almost all $x \in \Omega$, the Nemytskii operator $\Psi$ is continuous from $y_{M}$ to $L^{\infty}(\Omega)$ [Goldberg et al. 1992, Thm. 5]. Similarly,

$$
\psi_{t}:\left[-M_{y}, \infty\right) \times \Omega \rightarrow \mathbb{R}, \quad \psi_{t}(t, x):=\frac{-2 \beta}{(1+t)^{3}}
$$

is uniformly continuous for all $x \in \Omega$ and hence generates a continuous Nemytskii operator from $y_{M}$ to $L^{\infty}(\Omega)$. By Theorem 7 of [Goldberg et al. 1992], $\Psi$ is therefore continuously Fréchet-differentiable from $y_{M}$ to $L^{\infty}(\Omega)$ with derivative

$$
\Psi^{\prime}: y(x) \mapsto \frac{-2 \beta}{(1+y(x))^{3}} .
$$

Using this, it is straightforward to verify that $G$ is continuously Fréchet-differentiable (and hence strictly differentiable) with respect to $y$ for all $(\bar{u}, \bar{y}) \in \mathcal{U} \times y_{M}$, with Fréchet-derivative in direction $h \in y$ given by

$$
G_{y}(\bar{u}, \bar{y}) h=-\Delta h+\frac{-2 \beta \bar{u}}{(1+\bar{y})^{3}} h .
$$

We next argue surjectivity of $G_{y}$ on $L^{2}(\Omega)$, i.e., well-posedness of the linearized state equation (2.5) at $\bar{u} \in \mathcal{U}, \bar{y} \in \mathcal{y}_{M}$. We assume that

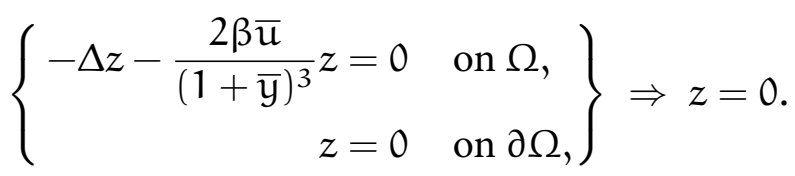


Note that condition (3.1) does not imply smallness of $\bar{u}$ (as is required for Lemma 2.5). This condition is equivalent to the condition that 1 is not an eigenvalue of the operator $A: L^{2}(\Omega) \rightarrow$ $\mathrm{L}^{2}(\Omega)$ defined by $\mathrm{A} z=(-\Delta+\mathrm{cI})^{-1} \mathrm{~b} z$, where $-\Delta+\mathrm{cI}$ is to be understood with homogeneous Dirichlet boundary conditions and $a=\frac{2 \beta \bar{u}}{(1+\bar{y})^{3}}, b=\max \{a, 0\}, c=\max \{-a, 0\}=-a+b$. Hence, this conditiona can be expected to be satisfied except in pathological situations. Note that for $\mathrm{n} \leqslant 3$, the operator $A$ is compact due to the estimate

$$
\|\mathrm{A} z\|_{\mathrm{H}^{\epsilon}(\Omega)} \leqslant \mathrm{C}\|\mathrm{b} z\|_{\mathrm{H}^{-(2-\epsilon)}(\Omega)} \leqslant \mathrm{C}\|\mathrm{b}\|_{\mathrm{L}^{2}(\Omega)}\|z\|_{\mathrm{L}^{2}(\Omega)}
$$

for some $0<\epsilon<2-\frac{\mathfrak{n}}{2}$.

We first show well-posedness and regularity of the linearized state equation.

Lemma 3.1. Let $\Omega \subseteq \mathbb{R}^{n}$ be bounded, and let (3.1) hold. Then for all $\bar{u} \in \mathcal{U}, \bar{y} \in y_{M}$ and $f \in \mathrm{L}^{2}(\Omega)$, the linearized state equation (2.5) has a unique solution $\mathrm{y} \in \mathrm{y}$.

Likewise, there exists $\mathrm{p}>\mathrm{n}$ such that for all $\mathrm{f} \in \mathrm{W}^{-1, \mathrm{p}}(\Omega)$, the linearized state equation (2.5) has a unique solution $\mathrm{y} \in \mathrm{W}^{1, \mathrm{p}}(\Omega)$, and there exist constants $\mathrm{c}, \mathrm{C}>0$ such that for all $\mathrm{f} \in \mathrm{W}^{-1, \mathrm{p}}(\Omega)$, the estimate

$$
c\|f\|_{W^{-1, p}(\Omega)} \leqslant\|y\|_{W^{1, q}(\Omega)} \leqslant C\|f\|_{W^{-1, p}(\Omega)}
$$

holds.

Proof. Again, since the coefficient of the zero-order term does not have the right sign and is not in $L^{\infty}(\Omega)$, we cannot apply standard results. In case $f \in L^{2}(\Omega)$, consider the compact operator $\tilde{\mathrm{T}}: \mathrm{C}_{0}(\Omega) \rightarrow \mathrm{C}_{0}(\Omega)$ defined by $\tilde{\mathrm{T}} v=(-\Delta)^{-1}(\mathrm{a} v)$, where $-\Delta$ is to be understood with homogeneous Dirichlet boundary conditions, and $a=\frac{2 \beta \bar{u}}{(1+\bar{y})^{3}}$. By assumption (3.1), the operator I $-\tilde{T}$ has Riesz index one. For any $f \in \mathrm{L}^{2}(\Omega)$, we have $(-\Delta)^{-1} \mathrm{f} \in \mathrm{C}_{0}(\Omega)$. Hence, (2.5) is equivalent to $(I-\tilde{T}) y=(-\Delta)^{-1} f$, which implies that $(2.5)$ has a unique solution in $y$.

Analogously, for appropriate $p$ we obtain unique solvability of $(2.5)$ in $\mathrm{W}^{1, p}(\Omega)$ for any $f \in \mathrm{W}^{-1, p}(\Omega)$ by considering the operator $\tilde{T}$ defined as above as a mapping $\tilde{\mathrm{T}}: \mathrm{W}^{1, p}(\Omega) \rightarrow$ $\mathrm{W}^{1, \mathrm{p}}(\Omega)$. For this purpose, we estimate

$$
\begin{aligned}
\|\tilde{T} v\|_{W^{s, p}(\Omega)} & \leqslant C\|\mathrm{a} v\|_{W^{-(2-s), p}(\Omega)}=C \sup \left\{\int_{\Omega} \varphi \mathrm{a} v \mathrm{~d} x: \varphi \in \mathrm{C}_{0}^{\infty}(\Omega),\|\varphi\|_{W^{2-s, p^{\prime}}(\Omega)}=1\right\} \\
& \leqslant C \sup \left\{\|\mathrm{a}\|_{\mathrm{L}^{2}(\Omega)}\|\varphi\|_{\mathrm{L}^{2 r /(r-1)}(\Omega)}\|v\|_{\mathrm{L}^{2 r}(\Omega)}: \varphi \in \mathrm{C}_{0}^{\infty}(\Omega),\|\varphi\|_{W^{2-s, p^{\prime}(\Omega)}}=1\right\} \\
& \leqslant \mathrm{C}\|\mathrm{a}\|_{\mathrm{L}^{2}(\Omega)}\|v\|_{\mathrm{W}^{1, p}(\Omega)},
\end{aligned}
$$

where $\mathrm{p}^{\prime}=\frac{\mathrm{p}}{\mathrm{p}-1}, \mathrm{C}>0$ denotes a generic constant, and we have used the Sobolev embeddings $\mathrm{W}^{2-s, \mathrm{p}^{\prime}}(\Omega) \hookrightarrow \mathrm{L}^{2 \mathrm{r} /(\mathrm{r}-1)}(\Omega)$ and $\mathrm{W}^{1, \mathrm{p}}(\Omega) \hookrightarrow \mathrm{L}^{2 \mathrm{r}}(\Omega)$ with appropriately chosen $\mathrm{r} \in[1, \infty]$ and $s \in(1,2]$. Indeed, the inequalities required for continuity of these embeddings, namely

$$
2-s+\frac{n}{p} \geqslant \frac{n}{2}+\frac{n}{2 r} \quad \text { and } \quad 1-\frac{n}{p} \geqslant-\frac{n}{2 r}
$$


can be satisfied by the following choices:

$$
\begin{array}{lll}
n=1: & p \in[1, \infty]: & r=2, s=\frac{5}{4}, \\
n=2: & p \in[1, \infty): & r=\frac{p+1}{2}, s=1+\frac{2}{p(p+1)}, \\
n=3: & p \in[1,6): & r=\frac{3 p}{6-p-\varepsilon}, s=1+\frac{\varepsilon}{2 p}, \varepsilon \in(\max \{0,6-4 p\}, \min \{6-p, 2 p\}) .
\end{array}
$$

(Note that by adding the two inequalities in (3.3), it becomes obvious that they cannot be satisfied for any $s>1$ as soon as $n \geqslant 4$.) Hence, $\tilde{T}$ is bounded as an operator from $W^{1, p}(\Omega)$ to $\mathrm{W}^{s, \mathrm{p}}(\Omega)$, and by the Rellich-Kondrachov theorem and boundedness of $\Omega$, compact as an operator from $\mathrm{W}^{1, p}(\Omega)$ to $\mathrm{W}^{1, p}(\Omega)$. By (3.1), the operator I - $\tilde{\mathrm{T}}$ has Riesz index one and is therefore bijective by the third Riesz theorem (e.g., Theorem 3.3 in [Kress 1999]). Moreover, by the second Riesz theorem (e.g., Theorem 3.2 in [Kress 1999]) it has closed range, hence by the Open mapping theorem (e.g., Theorem 2.11 in [Rudin 1991]) its inverse $(\mathrm{I}-\tilde{\mathrm{T}})^{-1}: \mathrm{W}^{1, \mathrm{p}}(\Omega) \rightarrow \mathrm{W}^{1, \mathrm{p}}(\Omega)$ is bounded. Using the fact that $(2.5)$ is equivalent to $(\mathrm{I}-\tilde{\mathrm{T}}) \mathrm{y}=(-\Delta)^{-1} \mathrm{f}$, we conclude estimate (3.2).

For the derivation of the optimality system, we also require the well-posedness of the adjoint problem with measure-valued right hand side. We follow the standard approach [Stampacchia 1965] (see also [Meyer et al. 2011]) and conclude from the fact that the linearized state equation operator is an isomorphism from $\mathrm{W}^{1, p}(\Omega)$ to $\mathrm{W}^{-1, p}$ that its adjoint is an isomorphism from $\mathrm{W}^{1, \mathrm{q}}(\Omega)$ to $\mathrm{W}^{-1, \mathrm{q}}$ with $1 / \mathrm{q}+1 / \mathrm{p}=1$. Due to the dense embedding $\mathrm{W}^{1, \mathrm{p}} \hookrightarrow \mathrm{C}_{0}(\Omega)$ for $\mathrm{p}>\mathrm{n}$, we have $\mathcal{M}(\Omega) \hookrightarrow \mathrm{W}^{-1, \mathrm{q}}(\Omega)$ for $\mathrm{q}<\frac{\mathrm{n}}{\mathrm{n}-1}$, and we deduce well-posedness of the adjoint equation.

Corollary 3.2. Let (3.1) hold. For any $\bar{u} \in \mathcal{U}, \bar{y} \in y_{M}, \mu \in \mathcal{M}(\Omega)$, and $q<\frac{n}{n-1}$, there exists a unique $\mathrm{p} \in \mathrm{W}^{1, \mathrm{q}}(\Omega)$, solving

$$
\left\{\begin{aligned}
-\Delta p-\frac{2 \beta \bar{u}}{(1+\bar{y})^{3}} p=\mu & & \text { on } \Omega, \\
p=0 & & \text { on } \partial \Omega .
\end{aligned}\right.
$$

Furthermore, there exists a $\mathrm{C}>0$ such that for all $\mu \in \mathcal{M}(\Omega)$, there holds

$$
\|p\|_{W^{1, q}(\Omega)} \leqslant C\|\mu\|_{\mathcal{M}(\Omega)} .
$$

\subsection{CONTROL PROBLEM}

The existence of minimizers follows again from standard arguments. For the sake of completeness, we nonetheless state the proof.

Theorem 3.3. There exists a minimizer $\left(u^{*}, y^{*}\right) \in \mathcal{U} \times y_{M}$ of $\left(\mathcal{P}_{s c}\right)$.

Proof. The set of feasible $(u, y)$ satisfying the equality and inequality constraints is non-empty (take $(u, y)=(0,0))$. By the boundedness of $y_{M}$ and the coercivity of the functional in $u$, we 
obtain the existence of a minimizing sequence which is bounded in $\mathcal{U} \times \mathcal{Y}_{M}$. Hence, there exists a subsequence, denoted by $\left(\left(u_{n}, y_{n}\right)\right)_{n \in \mathbb{N}}$, that weakly converges to $\left(u^{*}, y^{*}\right) \in \mathcal{U} \times y_{M}$. Due to the compact embedding of $y$ in $C_{0}(\Omega)$ and the continuity of $\Psi$, we have that a subsequence of $\frac{\beta}{\left(1+y_{n}\right)^{2}}$ converges strongly to $\frac{\beta}{\left(1+y^{*}\right)^{2}}$. Thus, we can pass to the limit in (the weak formulation of) $G\left(u_{n}, y_{n}\right)=0$ to obtain $G\left(u^{*}, y^{*}\right)=0$.

For the derivation of optimality conditions, we follow [Alibert and Raymond 1998]. Note that - as can be seen by inspection of the proofs in [Alibert and Raymond 1998] - it suffices to consider $G$ on the open set $\tilde{y}_{M}$ containing the admissible set, which is possible since $M_{y}$ is strictly less than 1 .

Theorem 3.4. Let $\left(\mathrm{u}^{*}, \mathrm{y}^{*}\right) \in \mathcal{U} \times \mathrm{y}_{\mathrm{M}}$ be a local minimizer of $\left(\mathcal{P}_{\mathrm{sc}}\right)$ and let (3.1) hold. Then there exist $\mu^{*} \in \mathcal{M}(\Omega)$ and $\mathrm{p}^{*} \in \mathrm{W}_{0}^{1, \mathrm{q}}(\Omega), \mathrm{q}<\frac{\mathrm{n}}{\mathrm{n}-1}$, satisfying

$$
\left\{\begin{aligned}
-\Delta p^{*}-\frac{2 \beta u^{*}}{\left(1+y^{*}\right)^{3}} p^{*} & =-\left(y^{*}-y_{d}\right)-\mu^{*}, \\
\alpha u^{*}-\frac{\beta}{\left(1+y^{*}\right)^{2}} p^{*} & =0, \\
\left\langle\mu^{*}, y-y^{*}\right\rangle_{\mathcal{M}, C} & \leqslant 0 \quad \text { for all } y \in y_{M} .
\end{aligned}\right.
$$

Proof. We recall that $\mathrm{G}$ is strictly differentiable at $\left(u^{*}, y^{*}\right)$. Furthermore, by Lemma 3.1, the linearized constraint $G_{y}\left(u^{*}, y^{*}\right)$ is surjective on $L^{2}(\Omega)$. Finally, a regular point condition is satisfied: There exists $\left(u_{0}, y_{0}\right) \in \mathcal{U} \times y_{M}$ such that $\left(y^{*}+y_{0}\right) \in \operatorname{int} y_{M}$ and

$$
G_{y}\left(u^{*}, y^{*}\right) y_{0}+G_{u}\left(u^{*}, y^{*}\right)\left(u_{0}-u^{*}\right)=0 .
$$

Specifically, if $y^{*} \in \operatorname{int} y_{M}$, take $\left(u_{0}, y_{0}\right)=\left(u^{*}, 0\right)$, otherwise take

$$
y_{0}=-\frac{M_{y}}{2 \sup _{x \in \Omega} y^{*}(x)} y^{*} \in y_{M}
$$

and

$$
u_{0}=u^{*}-\frac{M_{y}}{2 \sup _{x \in \Omega} y^{*}(x)}\left(\frac{\left(1+y^{*}\right)^{2}}{\beta} \Delta y^{*}+\frac{2 u^{*} y^{*}}{1+y^{*}}\right) \in \mathcal{U} .
$$

Using Corollary 3.2, we therefore obtain from Theorem 2.1 in [Alibert and Raymond 1998] the existence of unique Lagrange multipliers $p^{*} \in \mathrm{W}_{0}^{1, q}(\Omega)$ and $\mu^{*} \in \mathcal{M}(\Omega)$ corresponding to the equality and inequality constraint, respectively.

Remark 3.5. Existence of Lagrange multipliers $\mu^{*} \in \mathcal{M}(\Omega)$ and $p^{*} \in \mathrm{L}^{2}(\Omega)$ can be shown without the eigenvalue condition (3.1) by using a Maurer-Zowe constraint qualification [Maurer and Zowe 1979], which in our case amounts to solvability of

$$
\left\{\begin{array}{r}
-\Delta y^{*}+\frac{\beta u^{*}}{\left(1+y^{*}\right)^{2}}-\Delta y+\frac{\beta u}{\left(1+y^{*}\right)^{2}}-\frac{2 \beta u^{*}}{\left(1+y^{*}\right)^{3}} y=f \\
M_{y}+y^{*}+y-z=g
\end{array}\right.
$$




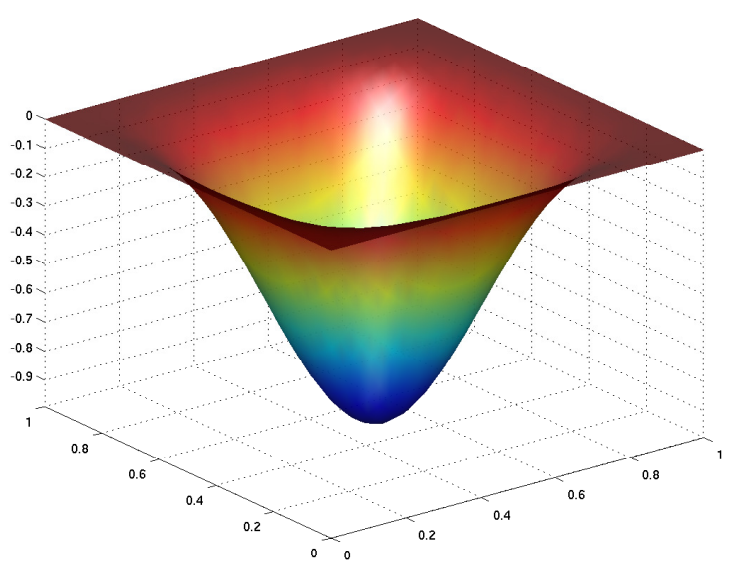

Figure 1: Target $y_{d}$

for any $(f, g) \in L^{2}(\Omega) \times C_{0}(\Omega)$ sufficiently small, by appropriately chosen $y \in \mathcal{y}, u \in \mathcal{U}$, $z \in \mathrm{K}=\left\{v \in \mathrm{C}_{0}(\Omega): v(x) \geqslant 0, x \in \bar{\Omega}\right\}$. Indeed, if $\|\mathrm{g}\|_{\mathrm{C}(\Omega)} \leqslant M_{y}$, by taking $y=-y^{*}$ and $z=M_{y}-g$ we can satisfy the second equation in (3.5). Solving the first one for $u$, we obtain a solution $(u, y, z) \in \mathcal{U} \times y \times K$. This implies existence of $\mu^{*} \in K^{+}, p^{*} \in L^{2}(\Omega)^{*}=L^{2}(\Omega)$ such that (3.4) holds, where the first line has to be understood in a very weak sense. However, higher regularity $\mathrm{p}^{*} \in \mathrm{W}_{0}^{1, \mathrm{q}}(\Omega)$, e.g., using Corollary 3.2, still requires condition (3.1).

\section{NUMERICAL EXAMPLES}

We illustrate the significant difference between the two approaches for a simple two-dimensional example where the target is far away from the set $W$. We set $\Omega=(0,1)^{2}$ and $\beta=1$. For the case of control constraints we set $M_{\mathfrak{u}}=0.14815$ according to (2.4); for the state-constrained problem we used $M_{y}=0.99$. The common target $y_{d}$ (shown in Figure 1 ) is chosen to attain the bound $\min _{x \in \Omega} y_{d}(x)=-M_{y}$ by setting

$$
y_{d}\left(x_{1}, x_{2}\right)=M_{y} \frac{\left(1+\cos \left(2 \pi x_{1}+\pi\right)\right)\left(1+\cos \left(2 \pi x_{1}+\pi\right)\right)}{4} .
$$

The penalty parameter was set to $\alpha=10^{-6}$ in both cases. The differential operators were discretized using second order finite differences on a mesh with $64 \times 64$ grid points.

To ensure feasibility of the iterates, the minimizers of $\left(\mathcal{P}_{c c}^{\prime}\right)$ and $\left(\mathcal{P}_{s c}\right)$ were computed using an interior point method (fmincon in MATLAB). In both cases, the final iterates attain the constraints and satisfy the optimality conditions to within a specified tolerance of $10^{-6}$.

The resulting optimal controls and states are given in Figures 2 and 3, while Figure 4 shows a cross-section at $x_{1}=0.5$ of $y_{d}, y_{c c}$ and $y_{s c}$. It can be observed that the control constraint leads to a very smooth optimal control, and that the smallness condition on the control is very restrictive and prevents attainment of the target. On the other hand, the state constraint allows much richer behavior of the optimal control and hence a significantly closer attainment. 


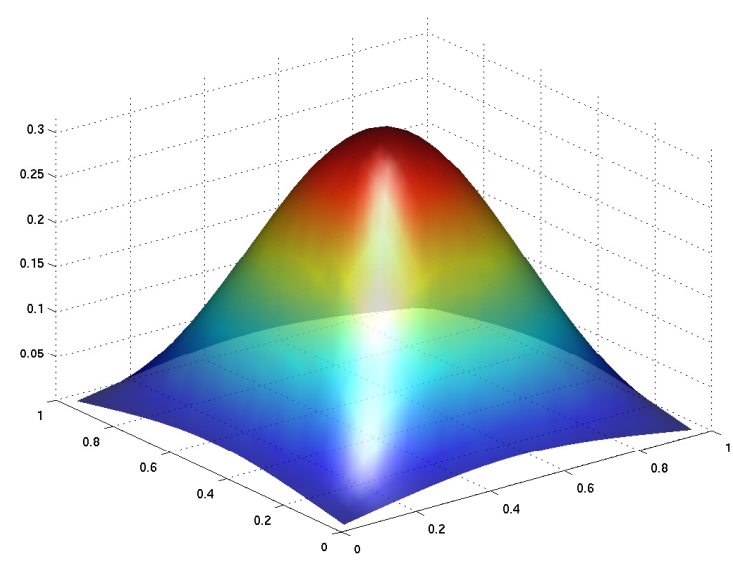

(a) optimal control $u_{c c}$

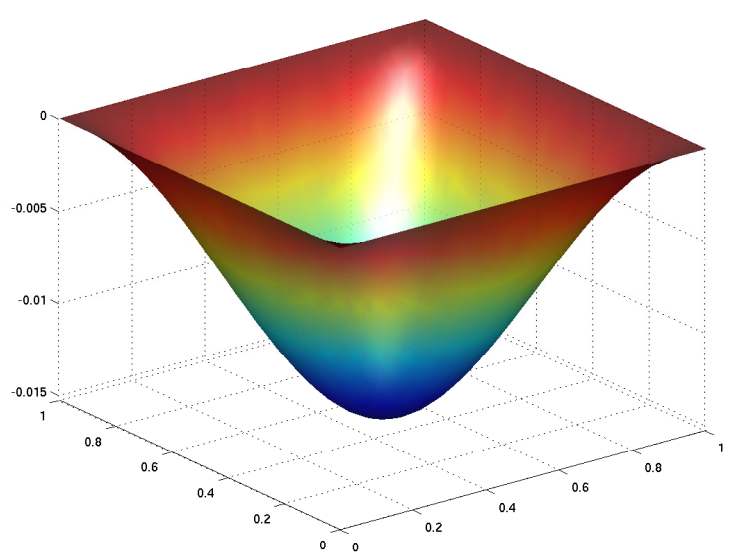

(b) optimal state $y_{c c}$

Figure 2: Solutions to control-constrained problem $\left(\mathcal{P}_{\mathrm{cc}}^{\prime}\right)$

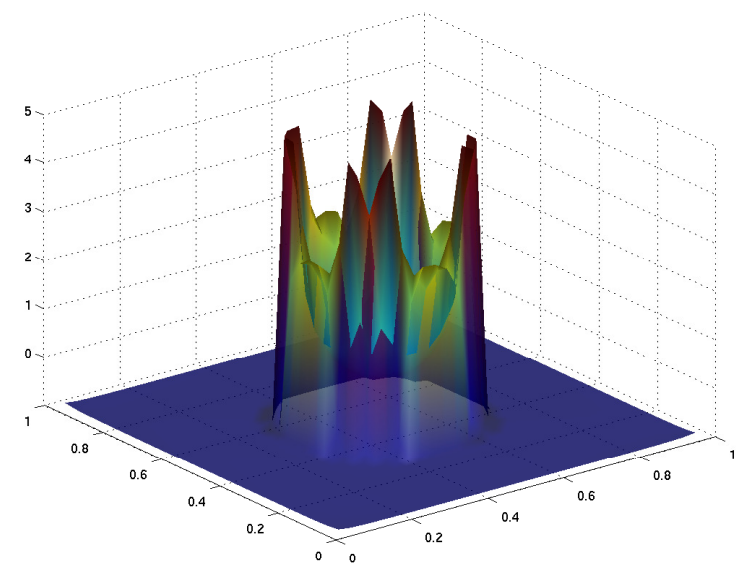

(a) optimal control $u_{s c}$

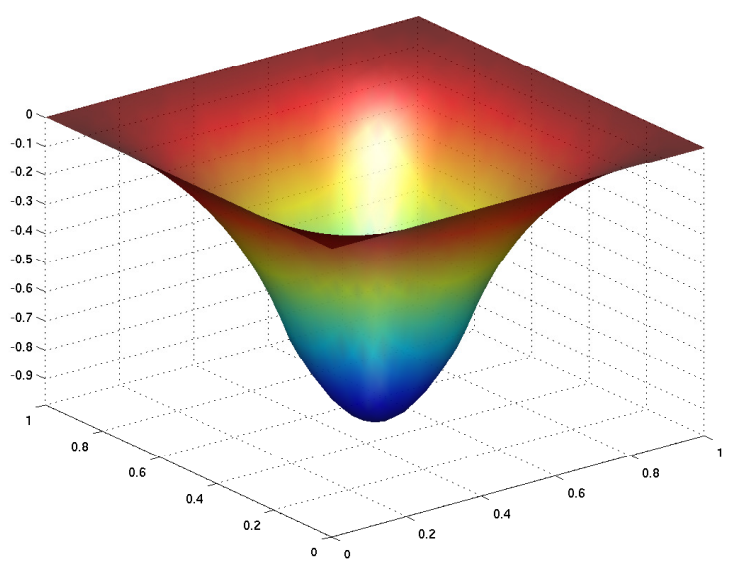

(b) optimal state $y_{s c}$

Figure 3: Solutions to state-constrained problem $\left(\mathcal{P}_{\text {sc }}\right)$ 


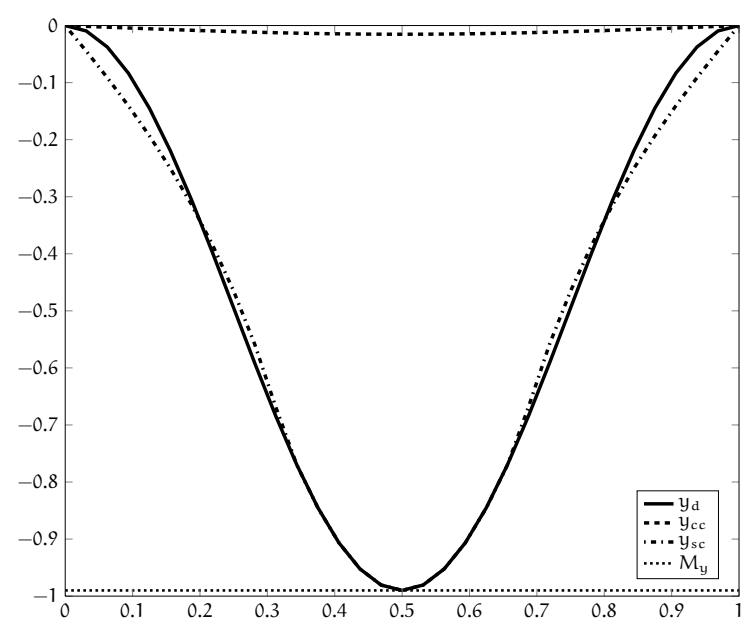

Figure 4: Cross sections of states for controlconstrained $\left(y_{c c}\right)$ and stateconstrained $\left(y_{s c}\right)$ problems, as well as target $y_{d}$ and bound $M_{y}$

\section{CONCLUSION}

Blow-up in optimal control of singular partial differential equations can be avoided by imposing suitable constraints on either the admissible controls or the admissible states. While the former allows applying standard techniques (via the introduction of the control-to-state mapping) to obtain existence and optimality conditions, the numerical examples for our model problem indicate that the required norm bounds can be overly pessimistic and prohibit even approximate attainment of the target. This indicates that state constraints are the more appropriate choice, even though they are computationally more involved and the constraint qualifications are easier to verify in the control-constrained case. In addition, the key requirements for deriving optimality conditions (via surjectivity of the linearized constraint) are less restrictive for state constraints. The suggested approach is expected to be similarly advantageous in many practically relevant applications involving the control of potentially singular or degenerate equations.

\section{ACKNOWLEDGMENTS}

The authors are grateful to the anonymous referees for their constructive comments which have led to an improved presentation. The work of the first-named author was supported by the Austrian Science Fund (FWF) under grant SFB F32 (SFB "Mathematical Optimization and Applications in Biomedical Sciences"). The second-named author would like to thank the German Science Foundation (DFG) for financial support within the grant KA 1778/5-1 ("Adaptive Discretization Methods for the Regularization of Inverse Problems"). 


\section{REFERENCES}

J.-J. Alibert and J.-P. Raymond (1998). A Lagrange multiplier theorem for control problems with state constraints. Numer. Funct. Anal. Optim. 19.7-8, pp. 697-704. D O I: 10. 1080/ 01630569808816854.

M. Bergounioux and K. Kunisch (1997). Augmented Lagrangian techniques for elliptic state constrained optimal control problems. SIAM J. Control Optim. 35.5, pp. 1524-1543. D O I: 10.1137/S036301299529330X.

J. F. Bonnans and A. Shapiro (2000). Perturbation analysis of optimization problems. SpringerVerlag, New York.

J. F. Bonnans and E. Casas (1989). Optimal Control of Semilinear Multistate Systems with State Constraints. SIAM Journal on Control and Optimization 27.2, pp. 446-455. D OI: $10.1137 / 0327023$.

E. Casas (1986). Control of an elliptic problem with pointwise state constraints. SIAM J. Control Optim. 24.6, pp. 1309-1318. D or: 10.1137/0324078.

G.-Q. G. Chen (2010). On degenerate partial differential equations. In: Nonlinear partial differential equations and hyperbolic wave phenomena. Amer. Math. Soc., Providence, RI, pp. 53-90.

C. Clason, B. Kaltenbacher, and S. Veljovic (2009). Boundary optimal control of the Westervelt and the Kuznetsov equation. Journal of Mathematical Analysis and Applications 356, pp. 738 751.

F. Colonius and K. Kunisch (1986). Stability for parameter estimation in two boundary value problems. Journal für Reine und Angewandte Mathematik 370, pp. 1-29.

H. Goldberg, W. Kampowsky, and F. Tröltzsch (1992). On Nemytskij operators in $\mathrm{L}_{p}$-spaces of abstract functions. Math. Nachr. 155, pp. 127-140. D o I: 10.1002/mana. 19921550110.

R. Kress (1999). Linear integral equations. Second. Vol. 82. Applied Mathematical Sciences. Springer-Verlag, New York. D O I: 10. 1007/978-1-4612-0559- 3.

J.-L. Lions (1985). Control of Distributed Singular Systems. Gauthier-Villars, Paris.

H. Maurer and J. Zowe (1979). First and second order necessary and sufficient optimality conditions for infinite-dimensional programming problems. Mathematical Programming 16, pp. 98-110.

C. Meyer, L. Panizzi, and A. Schiela (2011). Uniqueness Criteria for the Adjoint Equation in State-Constrained Elliptic Optimal Control. Numer. Funct. Anal. Optim. 32.9, pp. 983-1007. D OI: 10.1080/01630563.2011.587074.

J. A. Pelesko (2001/02). Mathematical modeling of electrostatic MEMS with tailored dielectric properties. SIAM J. Appl. Math. 62.3, pp. 888-908. D o I: 10. 1137/S0036139900381079.

J. A. Pelesko and D. H. Bernstein (2003). Modeling MEMS and NEMS. Chapman \& Hall/CRC, Boca Raton, FL. 
I. Pop and B. Schweizer (2011). Regularization schemes for degenerate Richards equations and outflow conditions. Mathematical Models and Methods in the Applied Sciences 21, pp. 1685-1712.

W. Rudin (1991). Functional analysis. Second. McGraw-Hill Inc., New York.

A. Schiela (2009). State constrained optimal control problems with states of low regularity. SIAM J. Control Optim. 48.4, pp. 2407-2432.

G. Stampacchia (1965). Le problème de Dirichlet pour les équations elliptiques du second ordre à coefficients discontinus. Ann. Inst. Fourier (Grenoble) 15.fasc. 1, pp. 189-258.

G. M. Troianiello (1987). Elliptic Differential Equations and Obstacle Problems. The University Series in Mathematics. Plenum Press, New York.

F. Tröltzsch (2010). Optimal control of partial differential equations. Vol. 112. Theory, methods and applications, Translated from the 2005 German original by Jürgen Sprekels. American Mathematical Society, Providence, RI. 